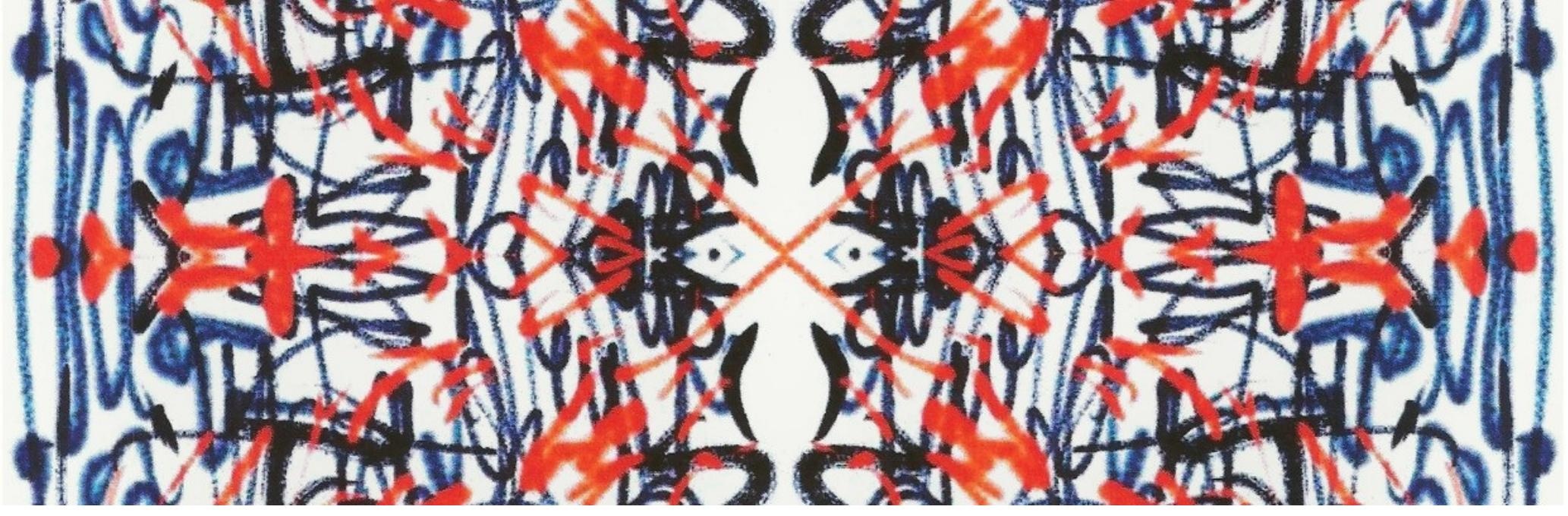

\title{
Definition and Usage of the Be-Bop Minor Scales (part 2): the Be-Bop Dorian Scale
}

Authors: $\quad$ Carmine Cataldo, Sandro Deidda, Francesco D’Errico, Giulio Martino

Submitted: $\quad$ 15. March 2018

Published: $\quad$ 25. March 2018

Volume: 5

Issue: 2

Affiliation: Independent Researcher, Jazz Pianist and Composer, PhD in Mechanical Engineering, Battipaglia (SA), Italy

Languages: Italian

Keywords: Jazz Improvisation, Be-Bop Dorian Scale, Minor Seventh Chords, Chordal Notes, Tensions, Approach Notes, Chromatisms, Harmonization, Arpeggios

DOI: $\quad$ 10.17160/josha.5.2.396

\section{JOSHA} josha.org
Journal of Science,

Humanities and Arts

JOSHA is a service that helps scholars, researchers, and students discover, use, and build upon a wide range of content 


\title{
Definizione ed Utilizzo delle Scale Minori Be-Bop (parte 2): la Scala Dorica Be-Bop
}

\author{
Carmine Cataldo*, Sandro Deidda**, Francesco D'Errico**, Giulio Martino** \\ *Jazz Pianist and Composer, PhD in Mechanical Engineering, Battipaglia (SA), Italy \\ **Department of Jazz Music, State Conservatory of Salerno, Salerno, Italy
}

\begin{abstract}
English)
In this article, the purpose of which may be regarded as solely didactic, some simple strategies finalized to mastering the Be-Bop Dorian scale are suggested. Moreover, their application to jazz improvisation is herein discussed. We start from the mere definition of the scale, extended along two adjacent octaves, in ascending and descending motion. Subsequently, the metric characteristics of the scale are highlighted, proposing performance, in descending motion, beginning at the chordal notes (tonic, modal, dominant, subtonic). Moreover, by exploiting some artificis typical of the Be-Bop idiom (such as chromatisms and approaches), we show how the scale may preserve its metric peculiarities even if it is performed beginning at the remaining tensions. Lastly, by resorting to the concept of harmonization, we expound a simple method finalized to combining the Be-Bop Dorian scale with the diatonic seventh arpeggios derived from the corresponding Dorian Scale.
\end{abstract}

\section{Keywords (English)}

Jazz Improvisation, Be-Bop Dorian Scale, Minor Seventh Chords, Chordal Notes, Tensions, Approach Notes, Chromatisms, Harmonization, Arpeggios.

\footnotetext{
Abstract

In quest'articolo, dalle finalità squisitamente didattiche, vengono suggerite alcune semplici strategie di metabolizzazione della scala Dorica Be-Bop, discutendone l'utilizzo in ambito improvvisativo. Si parte dalla mera esposizione della scala, estesa lungo due ottave adiacenti nei moti ascendente e discendente. Successivamente, se ne evidenziano le caratteristiche metriche, proponendone l'esecuzione, in moto discendente, a partire dalle note cordali (tonica, modale, dominante, sottotonica). Sfruttando alcuni artifizi tipici dell'idioma Be-Bop (quali cromatismi ed approcci) si mostra, inoltre, come la scala possa preservare le proprie peculiarità metriche anche qualora eseguita a partire dai restanti gradi tensivi. Infine, avvalendosi del concetto di armonizzazione, si illustra una semplice metodologia finalizzata alla combinazione della scala Dorica Be-Bop con arpeggi di settima diatonici dedotti dalla scala Dorica corrispondente.
}

\section{Keywords}

Improvvisazione Jazz, Scala Dorica Be-Bop, Accordi di Seconda Specie, Note Cordali, Gradi Tensivi, Note d'Approccio, Cromatismi, Armonizzazione, Arpeggi. 


\section{BREVE INTRODUZIONE}

In ambito improvvisativo, al lavoro sulle note cordali pure (triadi), altrove brevemente presentato [1] [2] [3] [4] [5] [6], è indubbiamente affiancabile quello sulle scale. Il lavoro sulle note cordali, considerate nulla più che punti di equilibrio intorno ai quali dar luogo a continue fluttuazioni, è rivolto alla creazione d'un linguaggio assai articolato, pertanto tutto fuorché lineare. Di contro, il lavoro sulle scale, adoperate tal quali ovvero introducendo, con la dovuta parsimonia, artifizi tipici dell'idioma Be-Bop (quali approcci, semplici e misti, cromatismi, ed enclosure) [7] [8] [9] [10], conferisce al linguaggio innegabile linearità. Com'è agevole intendere, la padronanza di entrambe le metodologie improvvisative contribuisce sensibilmente ad incrementare la varietà del linguaggio: in altri termini, il musicista diviene libero di selezionare, di volta in volta, il grado di linearità da adoperare nell'esposizione del proprio messaggio musicale. [11]

Tra le scale adoperate dai jazzisti, qualora tenuti ad improvvisare su un accordo minore [12] [13] [14] [15] [16] [17], è annoverabile la cosiddetta Dorica Be-Bop.

\section{LA SCALA DORICA BE-BOP}

Si riporta la scala Dorica Be-Bop di $C$, nei moti ascendente e discendente, estesa lungo due ottave consecutive.

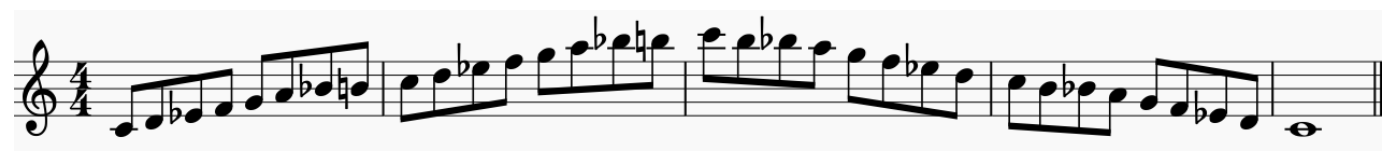

La scala appena presentata trova evidentemente applicazione in presenza dell'accordo $C-7$. [18]

L'aggiunta della settima maggiore (rispetto alla Dorica) è correlata all'ottenimento di un'interessante quadratura metrica. Eseguendo la scala a partire dalle note cordali (tonica, modale, dominante, sottotonica), queste risulteranno sempre posizionate in battere. Le tensioni residue (nona, undicesima, tredicesima) saranno ovviamente collocate in levare.

Uno dei primi esercizi in questa sede proposti, finalizzato ad una rapida metabolizzazione della scala, consiste nel suonare la Dorica Be-Bop in moto discendente, partendo dalle note cordali e raggiungendo, in ogni caso, la tonica (all'ottava inferiore rispetto a quella di partenza).

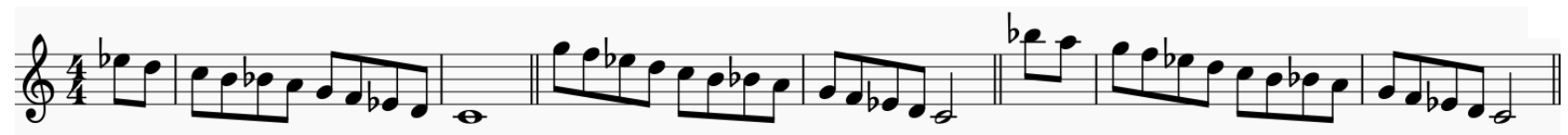

La frase con partenza dalla tonica è stata evidentemente omessa per brevità.

Per quanto affermato in precedenza, la quadratura metrica viene persa qualora la nota di partenza coincida con una delle tensioni. Il recupero metrico, tuttavia, è agevolmente ottenibile a mezzo di alcuni artifizi, assai diffusi nell'idioma Be-Bop. Il più banale consiste semplicemente nell'imporre un'anacrusi: in altri termini, la prima nota, che coincide con una delle tensioni residue, è suonata in levare. È inoltre possibile ribattere la tensione di partenza: coerentemente con la figurazione finora adoperata, pertanto, la frase inizierà, in tal caso, con due crome identiche consecutive. Alternativamente, è possibile raddoppiare la durata della tensione di partenza: sempre nel rispetto della figurazione adottata, la frase inizierà, in tal caso, con una semiminima. Inoltre, sono 
C. Cataldo, S. Deidda, F. D'Errico, G. Martino (2018)

contemplabili soluzioni, talvolta decisamente più interessanti, basate su cromatismi ed approcci misti (alle note cordali pure).

Proponiamo di seguito tre semplicissime frasi con partenza dalla sopratonica (ovvero dalla nona).

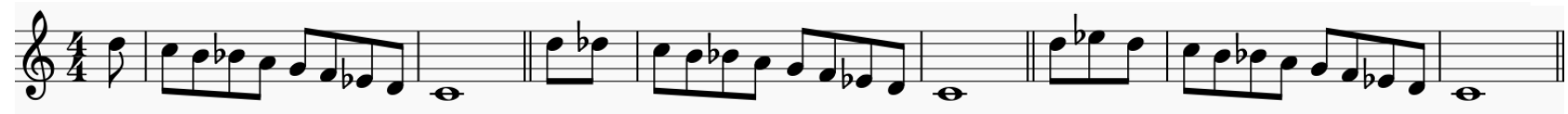

La prima è frase stata ottenuta imponendo un'anacrusi, la seconda costruendo (in anacrusi) un ponte cromatico verso la tonica, la terza imponendo (sempre in anacrusi, la cui durata è pari a $3 / 8$ al solo fine di collocare la tonica sul primo movimento dell'ultima battuta) un ponte cromatico verso la modale. A scopo squisitamente esercitativo, tutte le frasi proposte terminano sulla tonica (all'ottava inferiore rispetto a quella di partenza).

Proponiamo, adesso, tre semplicissime frasi con partenza dalla sottodominante (undicesima).

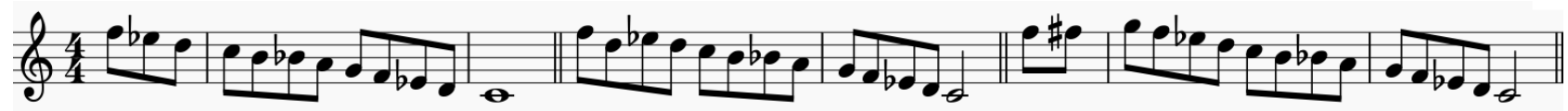

La prima frase è stata ottenuta imponendo un'anacrusi (la cui durata è pari a $3 / 8$ al solo fine di collocare la tonica sul primo movimento dell'ultima battuta), la seconda adoperando nulla più che un approccio misto alla modale (la sottodominante coincide con la nota d'approccio discendente alla modale), la terza costruendo (in anacrusi) un ponte cromatico verso la dominante. [19] Ancora una volta, le frasi terminano deliberatamente sulla tonica (all'ottava inferiore rispetto a quella di partenza).

Il secondo degli esempi sopra discussi ci offre l'opportunità di ricordare che, una volta definita la scala di riferimento, l'approccio alle note cordali pure (quelle costituenti la triade, essendo la settima soventemente qualificata come tensione fondamentale) è cromatico qualora ascendente, diatonico qualora discendente.

Proponiamo, infine, tre semplicissime frasi con partenza dalla sopradominante (sesta maggiore).

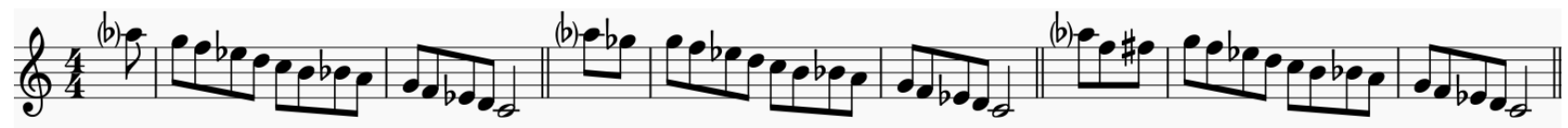

La prima frase è stata ottenuta imponendo un'anacrusi, la seconda adottando (in anacrusi) un approccio misto alla dominante, la terza adoperando (sempre in anacrusi, la cui durata è pari a $3 / 8$ al solo fine di collocare la tonica sul primo movimento dell'ultima battuta) un'enclosure [7] [8] [9] sulla dominante. Nuovamente, tutte le frasi sono intenzionalmente arrestate alla tonica. Il primo accidente in anacrusi, sebbene formalmente errato (nel rispetto della particolare scala considerata, l'approccio discendente alla dominante, diatonico per definizione, coincide con la sesta maggiore), viene spesso utilizzato al fine d'evitare la dissonanza tra settima minore (presente nel comping) e tredicesima. [19]

\section{COMBINARE SCALE E ARPEGGI}

Altro utilissimo esercizio consiste nell'esecuzione delle quadriadi terziane diatoniche deducibili dalla Scala Dorica. Nel caso particolare proposto in questa sede, considereremo accordi di settima 
C. Cataldo, S. Deidda, F. D'Errico, G. Martino (2018)

arpeggiati [20], in moto ascendente, dedotti dall'armonizzazione [21] [22] [23] della Doria di $C$ ovvero, equivalentemente, della Ionica di $B^{b}$.

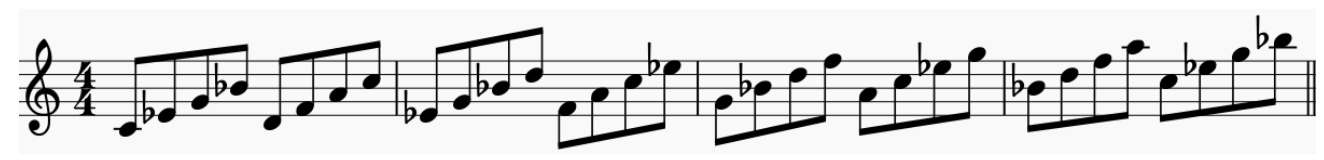

Le quadriadi terziane diatoniche possono essere agevolmente combinate con le frasi costruite sulla Dorica Be-Bop: molto semplicemente, l'ultima nota dell'arpeggio rappresenterà l'elemento di congiunzione tra il suddetto e la scala. A titolo d'esempio, la quarta nota dell'arpeggio ascendente di $E^{b}$ maj7 è $D$ (simultaneamente sensibile rispetto a $E^{b}$ e sopratonica rispetto a $C$ ): pertanto, il suddetto arpeggio potrà agganciarsi a qualsivoglia frase, costruita sulla Dorica Be-Bop, che preveda partenza dalla sopratonica (nona). Assai spesso, inoltre, si preferisce inserire un approccio ascendente (e quindi, per definizione, cromatico) alla prima nota dell'arpeggio. Proponiamo, di seguito, alcuni esempi (uno per ognuna delle note cordali) al fine d'illustrare qualitativamente il modo in cui le quadriadi terziane diatoniche possano combinarsi con la scala Dorica Be-Bop.
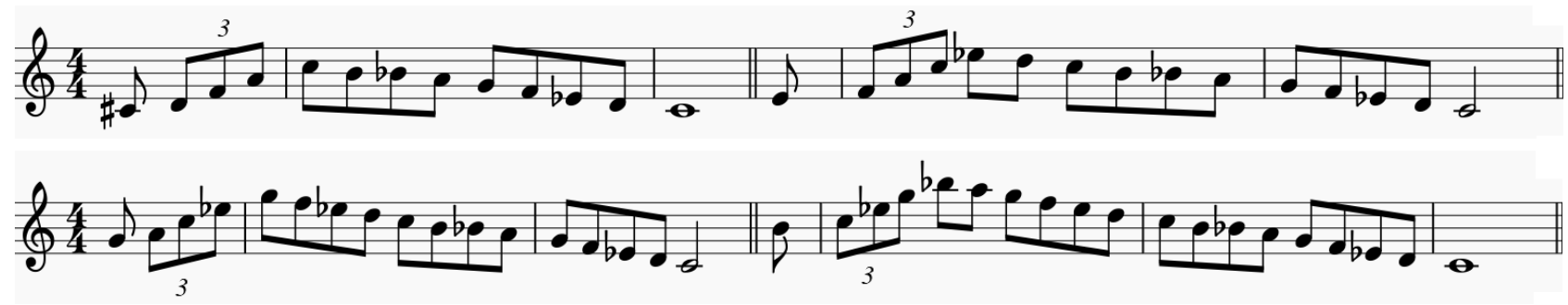

La prima nota dell'arpeggio è sempre approcciata. Le prime tre note costituiscono una terzina: l'adozione di tale gruppo irregolare, sebbene tutto fuorché strettamente necessaria, costituisce elemento distintivo dell'idioma Be-Bop. [19] Come sempre in questa sede, le frasi proposte terminano sulla tonica (all'ottava inferiore rispetto a quella di partenza). E d'uopo evidenziare come, al netto di quello costruito sulla sottotonica (settima minore), gli esempi appena riportati possiedano valore prevalentemente esercitativo.

Proponiamo alcuni esempi più articolati costruiti sulle tensioni (due per ognuna delle tensioni).
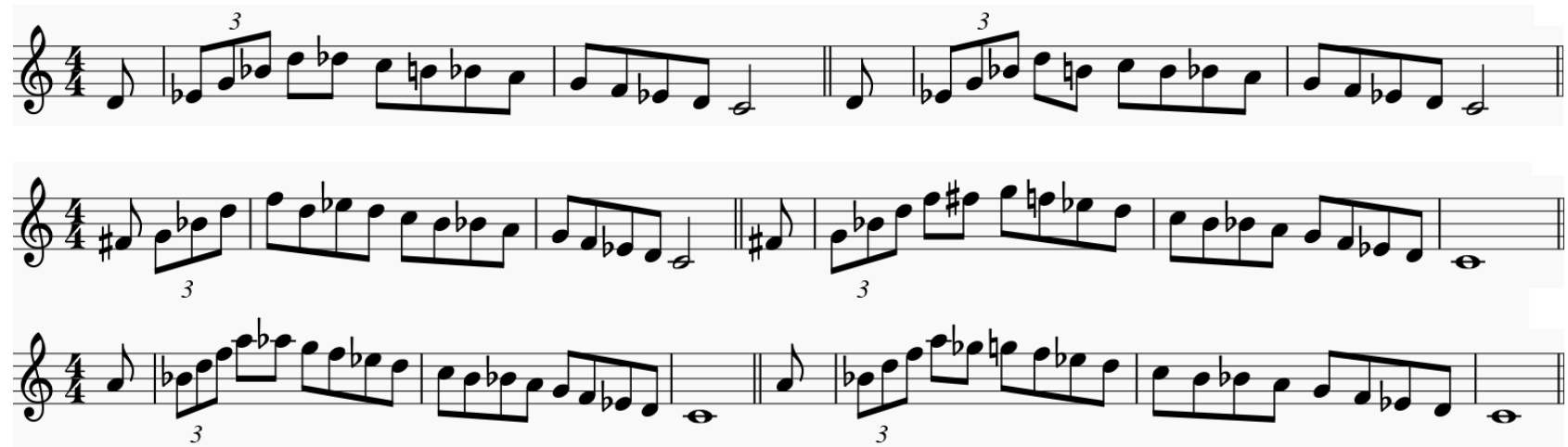

\section{OSSERVAZIONI FINALI}

A rigore, la scala Dorica Be-Bop propriamente intesa, sebbene di scarso utilizzo, prevede l'aggiunta della terza maggiore (rispetto alla Dorica) e non della sensibile (settima maggiore). Si riporta, di 
C. Cataldo, S. Deidda, F. D'Errico, G. Martino (2018)

Definizione ed Utilizzo delle Scale Minori Be-Bop (parte 2):

La Scala Dorica Be-Bop

Journal of Science, Humanities and Arts

seguito, la scala Dorica Be-Bop propriamente intesa di $C$, nei moti ascendente e discendente, estesa lungo due ottave consecutive.

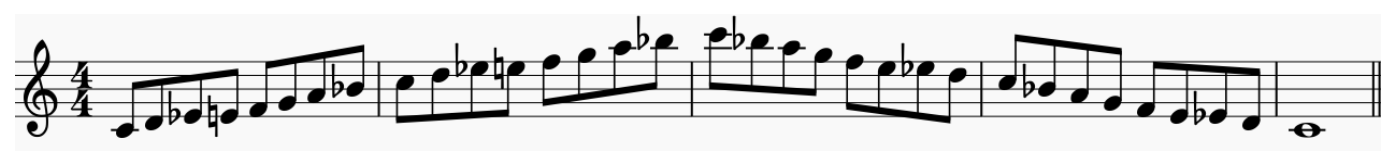

$\grave{E}$ agevole verificare come le note costituenti la scala appena presentata coincidano, nel caso specifico, con quelle della Dominante Be-Bop di $F$ : non a caso, in presenza di progressioni armoniche assai dense (quattro accordi per misura sulle ballad ovvero due sugli up-tempo), l'accordo $C-7$, seguito da F7, si contrae in quest'ultimo (dando luogo al fenomeno opposto a quello dell'espansione). [24] [25] Generalizzando, si può affermare come la Dorica Be-Bop propriamente intesa scaturisca dal quinto grado della Dominante Be-Bop (alternativamente, in termini forzatamente modali, il Dorico Be-Bop propriamente inteso può essere considerato come il quinto dei modi derivanti dalla Dominante Be-Bop).

Come altrove sottolineato, [26] [27] [28] la tentazione di sperimentare immediatamente più varianti in un'unica tonalità potrebbe risultare indubbiamente forte: tuttavia, lo studente dovrebbe evitare di cedere alle lusinghe di tale soluzione di comodo. Sarebbe fin da subito opportuno, infatti, affrontare lo studio nelle dodici tonalità procedendo, ad esempio, per quarte giuste ascendenti (ovvero, equivalentemente, per quinte giuste discendenti). Una prima fase dello studio potrebbe consistere nella mera esecuzione della scala; la seconda fase potrebbe essere dedicata alla riproduzione della scala partendo da ognuna delle note cordali pure e dalla sopradominante; nella terza, magari, si potrebbe rivolgere l'attenzione alle tensioni residue, e così via. In ognuna delle fasi, naturalmente, lo studio andrebbe condotto nelle dodici tonalità.

\section{RINGRAZIAMENTI}

Gli autori ringraziano l'amica Claudia Piscitelli per l'immagine di copertina.

\section{REFERENCES}

[1] Cataldo, C. (2017). The Art of Improvising: the Be-Bop Language and the Dominant Seventh Chords. Art and Design Review, 5, 181-188. http://doi.org/10.4236/adr.2017.53014

[2] Cataldo, C. (2017). Il Linguaggio Be-Bop e gli Accordi di Settima di Prima Specie [The Be-Bop Language and The Dominant Seventh Chords]. Journal of Science, Humanities and Arts (JOSHA), 4(4). https://dx.doi.org/10.17160/josha.4.4.340

[3] Cataldo, C. (2017). The Art of Improvising: the Be-Bop Language and the Minor Seventh Chords. Art and Design Review, 5, 213-221. https://doi.org/10.4236/adr.2017.54017

[4] Cataldo, C. (2017). Il Linguaggio Be-Bop e gli Accordi di Settima di Seconda Specie [The Be-Bop Language and The Minor Seventh Chords]. Journal of Science, Humanities and Arts (JOSHA), 4(4). https://dx.doi.org/10.17160/josha.4.4.339

[5] Cataldo, C. (2017). The Art of Improvising: the Be-Bop Language and the Major Seventh Chords. Art and Design Review, 5, 222-229. https://doi.org/10.4236/adr.2017.54018

[6] Cataldo, C. (2017). Il Linguaggio Be-Bop e gli Accordi di Settima di Quarta Specie [The Be-Bop Language and The Major Seventh Chords]. Journal of Science, Humanities and Arts (JOSHA), 4(4). https://dx.doi.org/10.17160/josha.4.4.341

[7] Baker, D. (1988). How to Play Bebop (Volume 1). Los Angeles, CA: Alfred Publishing Co. Inc.

[8] Baker, D. (1988). How to Play Bebop (Volume 2). Los Angeles, CA: Alfred Publishing Co. Inc.

[9] Baker, D. (1988). How to Play Bebop (Volume 3). Los Angeles, CA: Alfred Publishing Co. Inc. 
[10] Baker, D. (1988). Jazz Improvisation. Los Angeles, CA: Alfred Publishing Co. Inc.

[11] D’Errico, F. (2015). Fuor di Metafora - Sette Osservazioni sull'Improvvisazione Musicale. Naples, Italy: Editoriale Scientifica.

[12] Garland, R. (1999). The Jazz Piano Solos of Red Garland (by Tony Genge). Houston, TX: Houston Publishing.

[13] Kelly, W. (2013). The Wynton Kelly Collection: 25 Solo Transcriptions (by Michael Miller). New Albany, IN: Jamey Aebersold Jazz.

[14] Nelson, O. (2010). Patterns for Improvisation. New Albany, IN Jamey Aebersold Jazz.

[15] Parker. C. (1978). Charlie Parker Omnibook. Los Angeles, CA: Atlantic Music Corporation.

[16] Powell, B. (1998), Bud Powell Classics (Artist Transcriptions). Milwaukee, WI: Hal · Leonard.

[17] Powell, B. (2002). The Bud Powell Collection: Piano Transcriptions (Artist Transcriptions). Milwaukee, WI: Hal -Leonard.

[18] Levine, M. (2009). The Jazz Theory Book (Italian Edition by F. Jegher). Milan, IT: Curci Jazz.

[19] Wise. L. (1983). Bebop Bible - The Musicians Dictionary of Melodic Lines. United States: REH Publications.

[20] Coker, J., Casale, J., \& Campbell, G. (1982). Patterns for Jazz. Los Angeles, CA: Alfred Publishing Co. Inc.

[21] Cataldo, C. (2018). A Simplified Introduction to Music Algebra: from the Scale Vectors to the Modal Tensor. International Journal of Advanced Engineering Research and Science, 5(1), 111-113. https://dx.doi.org/10.22161/ijaers.5.1.16

[22] Cataldo, C. (2018). Algebra Musicale: dai Vettori Scala al Tensore Modale - Music Algebra: from the Scale Vectors to the Modal Tensor. Journal of Science, Humanities and Arts (JOSHA), 5(1). https://dx.doi.org/10.17160/josha.5.1.383

[23] D’Errico, F. (2017). Armonia Funzionale e Modalità - Rudimenti per l'Improvvisazione a Indirizzo Jazzistico. Naples, Italy: Editoriale Scientifica.

[24] Cataldo, C. (2018). Towards a Music Algebra: Fundamental Harmonic Substitutions in Jazz. International Journal of Advanced Engineering Research and Science, 5(1), 52-57. https://dx.doi.org/10.22161/ijaers.5.1.9

[25] Cataldo, C. (2018). Jazz e Sostituzioni Armoniche: Verso un Nuovo Formalismo - Jazz and Harmonic Substitutions: Towards a New Formalism. Journal of Science, Humanities and Arts (JOSHA), 5(1). https://dx.doi.org/10.17160/josha.5.1.381

[26] Cataldo, C., Martino, G. (2018). La Scala Dominante Be-Bop: Definizione ed Utilizzo - The Be-Bop Dominant Scale: Definition and Usage. Journal of Science, Humanities and Arts (JOSHA), 5(2). https://dx.doi.org/10.17160/josha.5.2.389

[27] Cataldo, C., Martino, G. (2018). La Scala Maggiore Be-Bop: Definizione ed Utilizzo - The Be-Bop Major Scale: Definition and Usage. Journal of Science, Humanities and Arts (JOSHA), 5(2). https://dx.doi.org/10.17160/josha.5.2.393

[28] Cataldo, C., Deidda, S., D’Errico, F., Martino, G. (2018). Definition and Usage of the Be-Bop Minor Scales (part 1): the Be-Bop Melodic Minor. Journal of Science, Humanities and Arts (JOSHA), 5(2).

https://dx.doi.org/10.17160/josha.5.2.395 